\title{
La confianza de los jóvenes chilenos y su relación con la cohesión social ${ }^{1}$
}

\author{
Dr. Mario Sandoval
}

\author{
Yo confío \\ tú confías \\ él confía \\ nosotros confiamos \\ ellos confían \\ iiqué manga de ingenuos!! \\ MAFALDA
}

\section{Resumen}

En el presente artículo se presentan diversas concepciones de confianza, comenzando por mencionar que puede ser entendida como un proceso racional, el cual permite señalar que la confianza es concebida como un estado abstracto en los sujetos, que se desarrolla de forma consciente, es decir, las personas racionalizan la confianza que depositan en otros, por lo mismo ésta puede ser vista como un estado intelectivo del hombre que le indica seguridad y optimismo frente a su medio. La confianza constituye las relaciones sociales y está íntimamente ligada a la cohesión social. En ese proceso los jóvenes presentan altos grados de desconfianza en las instituciones sociales, en particular las instituciones relacionadas con la política, lo cual podría hipotetizar que esta baja confianza en las instituciones encargadas de los destinos colectivos atenta contra la posibilidad de generar adecuados grados de cohesión social.

Palabras clave: Juventud, confianza, cohesión social, sociedad

\begin{abstract}
Diverse conceptions of trust are presented in the present article, starting by mentioning that it can be understood as a rational process; conceived as an abstract state in people, which is developed consciously. This implies that people rationalize the trust that is bestowed on others, meaning that it can be seen as a state of mind of men which indicates security and optimism in his relationship with his environment. Trust builds social relationships and is strongly associated with social cohesion. In this process, young people are very wary of social institutions, in particular those related to politics. This phenomenon -the lack of trust in the institutions that are in charge of collective matters- would jeopardize the possibilities of achieving good levels of social cohesion.
\end{abstract}

Key words: Youth, trust, social cohesion, society

1 El presente artículo es producto del Proyecto FONDECYT N 1100649 “Vinculaciones entre la construcción y deconstrucción de la confianza y la cohesión social, en jóvenes estudiantes de educación secundaria: lineamientos para fortalecer la democracia". 


\section{Introducción}

Cuando la adecuación de la estructura social y la subjetividad de los individuos se relaja o se quiebra, la sociedad cede su lugar a la comunidad; es así como los diferentes autores sociológicos nos describen el declive de la sociedad industrial, de las sociedades nacionales y los ajustes que realizan los individuos en sus roles y conductas cuando los valores compartidos se esfuman o evaporan en el aire.

De esta manera surgen diversos análisis que nos plantean sociedades "post" que manifiestan la separación del sujeto con la sociedad, el descentramiento social producto de los cambios vertiginosos que se están produciendo en todos los niveles (social, religioso, político, económico, cultural, etc.).

Desde su fundación, la sociología se ha preocupado de analizar la división del trabajo social (Durkheim), los procesos de racionalización (Weber), el individualismo (Touraine) y la historia del capitalismo (Marx). En cada uno de estos constructos, surge la oposición integración-cohesión, como dos maneras de entender la sociedad.

Según F. Dubet (2009), la integración corresponde a la sociedad y la cohesión nos reenvía a las formas de vida social en las cuales estamos entrando. De esta manera, la cohesión social designa una manera de producir la sociedad; es menos un tipo-puro (al estilo de los ideales-tipo de Weber) que un deslizamiento en las maneras de definir los problemas sociales y sus soluciones.

La noción de cohesión social podría ser considerada como una trampa del neoliberalismo que apunta a considerar que la vida social no es sino una forma de mercado entre otras y que solo el criterio de juicio pertinente sería la eficacia económica.

Desde esta perspectiva, la cohesión social surge de sociedades individualistas que no imponen posiciones ni roles, sino más bien colocan pruebas a los individuos, pruebas que deben superar movilizándose ellos mismos, con el fin de lograr su autonomía, con el fin de ser sujetos. 
Así, cohesión social y confianza pasan a ser dos conceptos íntimamente relacionados que están en la base del funcionamiento social actual; es por eso que a continuación desarrollaremos ambos conceptos con el fin de elucidar los complejos mecanismos a través de los cuales los sujetos se construyen a sí mismos y de paso dan vida a la sociedad moderna.

\section{La confianza:}

La confianza es parte constitutiva de las relaciones sociales, más aún, la confianza facilita las relaciones sociales reales, las hace más efectivas y con permanencia en el tiempo. Su análisis muchas veces queda obviado, ya que todas las personas han tenido la experiencia vital de confiar, o al contrario, desconfiar, convirtiéndose en un término cercano que quizás no exige fundamentos.

Actualmente esta idea está cambiando, influyendo directamente a las sociedades. Pareciera ser que la confianza no se asimila como antes, o está siendo cuestionada por las nuevas generaciones. Revisar y sistematizar las principales acepciones sobre la confianza puede derivar en ingeniosas ideas de cómo preservarla y extenderla, con el fin de mantener un mundo estable y con futuro social.

La confianza es adquirida en las primeras etapas de la vida, al interior de la familia, fundada en los cuidados maternales, y al ser abordada desde la niñez se considerará que en las etapas posteriores, cuando los individuos comienzan a relacionarse con otros, podrán generar confianza social.

El proceso emotivo, alude a que la confianza es originada por razones afectivas o sentimientos propios del individuo, caracterizada por ser espontánea e impulsiva, se expresa a personas con las cuales nos involucramos afectivamente, es decir, hacia familiares, amigos y/o compañeros de trabajo.

Según Moreno la confianza se encuentra ligada a la personalidad del individuo, la cual puede ser definida basándose en el efecto exterior o en la estructura interna. 
Las definiciones basadas en el efecto exterior se refieren a la personalidad como "un conjunto de cualidades que, idealmente, resultan ser socialmente agradables y eficaces" y puede ser desarrollada reforzando el conjunto de cualidades que la componen (Allport, 1986 p. 41). Entonces la personalidad se observará en el modo de comportarse o de actuar e influirá sobre otras personas, por ende, los individuos emitirán juicios, generarán opiniones de otros y a partir de éstas decidirán si confiar o no (Allport, 1986).

La personalidad, considerada como estructura interna, pretende demostrar que ésta tiene historia y existencia propia y no debe confundirse con la sociedad ni con las percepciones que otros individuos tienen de otra persona.

La relación maternal de los primeros años puede no seguir desarrollándose en el período juvenil ya que en esta etapa se tiende al alejamiento parental y se refuerzan las relaciones entre pares.

En la última etapa vivida por los individuos, cabe esperar que la confianza se haya convertido en "la fe más madura de una persona que envejece pueda alcanzar en su marco cultural y su período histórico" (Erikson, 1987 p.244).

Para la psicología la confianza no solo se asienta y se queda en los estados de conciencia de los individuos, es decir, no solo se considera la perspectiva racional o afectiva, sino que la acción de confiar implica la capacidad del individuo de comprender e instaurar normas sociales, aprendidas en las primeras etapas de la vida, que permiten confiar en otros.

Desde esta perspectiva, la confianza se basa en la esperanza de una persona o grupo en poder contar con una promesa de otra persona o grupo, esta promesa puede ser expresada de forma escrita $\mathrm{u}$ oral y a su vez puede ser positiva o negativa (Petermann en Laso, 2007).

La confianza se encuentra relacionada con la esperanza que se tendrá en las palabras y acciones que la otra persona prome- 
te, entonces se deberá ver reforzada en función de las acciones, es decir, si un individuo mantiene aquello que ha permitido que se sepa acerca de él, ya sea consciente o inconscientemente será acreedor de confianza, porque mantiene una congruencia en su actuar que proporcionará credibilidad y confianza (Luhmman, 1996 pp. $65-66)$,

Cualquiera que confía tiene que estar preparado para aceptar los riesgos que implica, debe tener en claro que no está confiando incondicionalmente, sino más bien dentro de límites y en proporción a expectativas racionales y específicas (Luhmman, 1996).

Respecto a la confianza pública, las personas confiadas tienden a confiar más en el gobierno, en el Parlamento, la justicia, los partidos políticos y los sindicatos. Cuando se trata de confianza social las personas que confían se expresan a favor de la confianza hacia los amigos, los compañeros de trabajo y los vecinos (Valenzuela y Cousiño, 2000).

Si no existe confianza es posible que el sujeto se distancie de su realidad cotidiana, se va sintiendo ajeno a todo su entorno, lo cual fomentará la incapacidad de cumplir con lo prometido de forma continuada en el tiempo. Todas las personas buscan seguridad en la vida pero la mayoría de las personas desconfiadas tienden a hacerlo, cerrándose a nuevas posibilidades, reprimiéndose a colaborar y a participar en la sociedad (Montañés, s/f).

Si la vida en sociedad se vuelve individualista y más compleja es debido a la falta de lealtad y al individualismo egoísta, ya que estos deterioran las relaciones humanas, y entonces se instala la desconfianza, y ésta puede ser hacia el sistema y la política (desconfianza vertical) y/o desconfianza proveniente del déficit racional que ha surgido entre los ciudadanos -desconfianza horizontal- (Keim, 2001).

Para Moya (2001, p. 104), la confianza como expresión "denota el fenómeno del acompañar, del estar respecto del otro(a), ya sea de forma personal o social, que otorga fianza, que fía. Es 
el estar en disponibilidad del encuentro que acoge; lleva en sí el compromiso de lo social".

En este sentido, la acción confiada muestra conductas que aumentan la propia vulnerabilidad, tienen lugar frente a una persona no sujeta al control personal, se escogen en una situación en que el daño que posiblemente se padece es mayor que el provecho que se puede sacar de la conducta, en ese sentido la confianza se refiere a acciones futuras de otros, que eluden el propio control y por ello implican incerteza y riesgo. La confianza se muestra en la disposición a hablar de temas que potencialmente pueden provocar desaprobación y rechazo, por tanto presentan un riesgo (Laso, 2007).

La confianza social comprende el valor para otros y para la sociedad en general. Restaurar la confianza e incrementar la predisposición de cada individuo a sentirla, implica confiar en otras personas, lo cual comprende riesgos, aunque el riesgo es mucho mayor cuando no se confía; de esta manera, la confianza es parte integral del tejido de una sociedad, depende de ella. Se da por sentado que allí está, hasta que se contamina o destruye, entonces da cuenta de que la confianza es vital para el bienestar de las personas (Covey y Merrill, 2007).

La confianza social es la confianza en desconocidos acerca de los que se carece de información respecto de si son o no dignos de confianza. El misterio de la confianza social es que, si no se tiene información acerca del otro, no hay base para saber si es o no digno de confianza (Herreros, 2004).

Los actos declarativos de confianza se dan entre personas y se relacionan con la capacidad de concertarse y acordar horizontes comunes de vida; la confianza entre sujetos se vincula con personas que hablan, creen, piensan, sienten y se construye como una relación social, histórica y culturalmente cotidiana (Olea, 2001)

En este sentido, Martínez (2001, p. 60) plantea que "la confianza se funda y garantiza principalmente en una ética de la res- 
ponsabilidad individual, que descansa en el hecho básico de que toda persona cumple y respeta las promesas y compromisos que ha declarado frente a otros. Es la promesa mutua y su cumplimiento lo que asegura el éxito del vínculo con extraños. Sólo a través de la mantención de las promesas y de la palabra dada es posible la constitución de relaciones seguras y constantes entre personas que no se conocen".

En este sentido, existen tres ámbitos donde se puede confiar o no:

a) institucional,

b) interpersonal inter-grupos,

c) interpersonal intra-grupos.

Los tres tipos de confianza pueden apoyarse mutuamente o compensar sus deficiencias y fracasos. Además, este autor plantea que la asociatividad no fomenta la confianza social, sino que la debilita, pues es un intento de reducir el riesgo de la baja de confianza social construyendo grupos que aumenten la confianza específica.

Respecto de la confianza las sociedades enfrentan una encrucijada, puesto que o avanzan en dirección de la abstracción aumentando la confianza social y la tolerancia, o bien subsanan su ausencia acrecentando la confianza intragrupos, generando una sociedad cada vez más segmentada, dispersa, desigual e injusta; fortaleciendo las instituciones formales de control (Laso, 2007).

Los intereses inmediatos de cada individuo hacen ver la vida como una crónica del yo, lo cual tiene implicancias en el que el contacto con los acontecimientos y hechos se vincula más a la vida individual (Olea, 2001).

En relación al contexto chileno, Martínez (2001, p. 65), citando a Giddens, señala que "la sociedad chilena se niega a la posibilidad de reconocer-se, de auto-observarse, en definitiva de ser reflexiva". 
En Chile existe una gran distancia entre las declaraciones o discursos de los sujetos y sus acciones, es decir, entre valores declarados y sus comportamiento; esta situación aumenta cuando se trata de las clases sociales inferiores, las que legitiman mayormente este tipo de prácticas ante la percepción de injusticia social de la cual serían objeto (Martínez, 2001).

\section{- Tipos de confianza}

Robert Putnam presenta una distinción entre la confianza densa y la confianza diluida, la primera estaría basada en las relaciones personales fuertes, frecuentes y establecidas en unas redes más amplias, que involucran a un conjunto concreto de individuos del entorno, por consiguiente, es la confianza que depositamos en gente a la que conocemos muy bien, familiares y amigos. La confianza diluida se refiere al otro en general, se extiende más allá de aquellos a los que conocemos personalmente. La forma de confianza más diluida estaría configurada por lo que se suele denominar en la literatura confianza social generalizada, y consiste en conceder el beneficio de la duda a aquellos que no conocemos (Putnam, 2002, p. 178).

Para Putnam la confianza interpersonal se explicaría como parte de la confianza densa, ya que se aplica a gente a la que se conoce bien, con la que se tienen lazos familiares o de amistad.

Fukuyama considera la confianza como base del desarrollo en una sociedad y la confianza interpersonal como base para la constitución y construcción de capital social, sólo cuando está inmersa en una relación social. Por lo mismo, es importante hacer la conexión de la confianza interpersonal generalizada, aquella que se tienen entre los ciudadanos, con el capital social, ya que facilita la coordinación, cooperación y participación entre los individuos que aportará el beneficio mutuo (Putnam, 1994, en Kliksberg y Tomassini, 2000).

La confianza puede ser clasificada de acuerdo a los niveles donde se construye o a la escala que utiliza: existe en primer tér- 
mino la confianza social o generalizada, versus la confianza particularizada. Esta distinción la propone el informe de la CEPAL (2007), en el cual indica una definición para confianza social o generalizada similar a las ideas descritas anteriormente. Postula que la confianza social es la "creencia en que una persona o grupo será capaz y deseará actuar de manera adecuada en una determinada situación, lo cual supone un cierto grado de regularidad y predictibilidad de las acciones que facilitan el funcionamiento de la sociedad".

Señala la CEPAL que "un elemento básico para el desarrollo de la confianza interpersonal es la participación en organizaciones y asociaciones cuyos miembros solo comparten algunos intereses, es decir, en grupos de personas que tienen características heterogéneas" (p. 78), lo que llevará al aprendizaje sobre la confianza social, haciéndose extensivo este ejercicio de relaciones a los desconocidos (Herreros, en CEPAL, 2007).

T. Moulian (2001) especifica lo que es la confianza política, respecto a otras confianzas. Es esta la que "se adquiere cuando todas las partes tienen una certeza razonable de equidad política, de transparencia y accesibilidad (de acortamiento de la distancia arriba - abajo). El camino para ello es una democracia participativa, en la cual los ciudadanos no transfieren totalmente su poder soberano entre elección y elección (régimen presidencial) o entre adquisición y pérdida de la mayoría (régimen parlamentario). Esto se basa en una razón de fondo: la confianza relacional es una virtud pragmática, que no anula la desconfianza ontológica en el dominio estatal" (p. 36).

En la misma línea, García (2001) señala que hay dos formas en que se expresa la confianza política: "primero en sus instituciones y, segundo, en sus grados de adhesión al sistema democrático y en las respuestas de lealtad que tienen los ciudadanos hacia éste, medido por el factor de la desconfianza interpersonal" (p. 45). Las definiciones de estos dos autores, con sus especificaciones, ayudan al entendimiento de cómo la confianza se inserta en todos los niveles de la vida social, y asimismo, cómo las nociones tienen puntos de 
convergencia que orientan la práctica de la confianza hacia la obtención de virtudes político-sociales.

\section{- Confianza Interpersonal}

El riesgo y/o la incertidumbre que ya han sido mencionados, generarán que las personas inicien interacciones a partir de la desconfianza, manteniendo, en primer momento, distancia hacia otro y buscarán protegerse a sí mismos evitando ser muy impulsivas o rígidas al actuar, por lo tanto, mantendrán cierto control en el primer momento de interacción, compartirán cosas intrascendentes, superficiales, tomando precauciones respecto a lo que se comunica.

Si se logra disminuir la excesiva vigilancia, la preocupación y la rigidez, se podrá pasar a un segundo momento de interacción donde se comenzarán a compartir experiencias y vivencias más relevantes e íntimas (Montañés, s/f), lo cual da paso a una relación que resulta ser estable en el tiempo porque se comienza a generar condiciones para que surja confianza.

La confianza permite que dos personas o más, en un contexto íntimo, logren estar en la certeza de que el otro, cuya verdad y realidad son diferentes, permitirá ser lo que se desee respetando las diferencias.

La confianza interpersonal se basa en la suposición de que los otros tienen buenas intenciones para con nosotros, entonces si una persona realiza una promesa a otra y se confía que ésta se cumplirá, la confianza servirá para superar o reducir el elemento de incertidumbre en el comportamiento de otras personas.

Para reducir la incertidumbre y generar confianza interpersonal se debe cumplir con requisitos fundamentales como la sinceridad, transparencia y respeto, los cuales se observan principalmente en las relaciones que tienen los amigos, familiares y entorno más cercano.

Si hablamos de condiciones o requisitos se debe considerar 
que la responsabilidad es un valor importante para generar confianza, ya que la confianza que surge desde la responsabilidad, se entiende como la capacidad de una persona de cumplir sus compromisos con otros, en este sentido romper una promesa o no cumplir un compromiso repercute en la construcción o deconstrucción de confianza (Erikson, 1987).

La confianza interpersonal puede ser dividida en dos aspectos: confianza específica o intragrupos, confianza generalizada o intergrupos. Confianza específica o intragrupos, refiere a que se puede confiar en alguien porque es conocido y es parte del entorno, es decir, en aquellas personas con las cuales se mantiene un vínculo estrecho, por ende se comparten los mismos valores, siendo fácil conocer y entender sus comportamientos (Laso, 2007).

Confianza generalizada o intergrupo se refiere a que los individuos confían en personas extrañas, entonces se confiará en cualquier sujeto con el que entablamos una conversación o compartimos el mismo espacio físico (Laso, 2007). Esta confianza se desarrolla porque la persona considerará que no existen razones para desconfiar, ya que aún no se conocen las intenciones del otro sujeto.

La confianza en los chilenos:

Según los resultados de la Encuesta del Programa de Naciones Unidas para el Desarrollo (PNUD, 2000), los chilenos expresan los siguientes niveles de confianza en las instituciones.

$\begin{array}{lr}\text { Iglesia Católica } & 54,8 \% \\ \text { Universidades } & 33,3 \% \\ \text { Constitución Política } & 6,4 \% \\ \text { Parlamento } & 3,6 \% \\ \text { Partidos Políticos } & 1,7 \%\end{array}$

Según el mismo estudio, en el año 2006 se observaron los siguientes datos

$\begin{array}{ll}\text { Gobierno } & 4,5 \% \\ \text { Policía } & 4,9 \% \\ \text { Partidos Políticos } & 3,3 \%\end{array}$


Los porcentajes de confianza entregados por la misma Encuesta el año 2008 fueron los siguientes:

$\begin{array}{ll}\text { Gobierno } & 4,4 \% \\ \text { Policía } & 5,0 \% \\ \text { Partidos Políticos } & 3,4 \%\end{array}$

Si se contrastan estos datos con otros países de Latinoamérica, Chile se ubica más abajo que Uruguay y México.

Respecto a la confianza entre las personas, los chilenos confían en el prójimo en un 14\% y desconfían en un 87\%. Comparado con Europa Occidental los niveles de confianza en el prójimo son superiores a los que se aprecian en Chile, por ejemplo, en Suecia la confianza asciende a un $66 \%$, Holanda 53\%, Alemania 38\%, España 36\% (Valenzuela \& Cousiño, 2000).

A su vez el PNUD señala que el 63,3\% desconfía de las personas y sólo un $32,4 \%$ manifiesta confianza en sus semejantes (PNUD, 2000).

Estos datos nos permiten inferir que en Chile se confía mayormente en aquellas personas que son conocidas, como familiares y amigos, pero no en personas externas al mundo cotidiano, lo cual dificulta la cohesión social.

La Encuesta sobre asociatividad realizada por la Dirección de Estudios Sociológicos de la Pontificia Universidad Católica de Chile, que analizó el contraste entre las tasas de confianza social que muestran Chile y Estados Unidos según resultados de encuestas comparables entre ambos países, sostiene que la disposición a confiar en los demás está en el origen de la aptitud típica de los habitantes de Estados Unidos para establecer relaciones sociales con desconocidos, que se expresa en la fortaleza de sus relaciones de amistad. En cambio, la sociedad chilena se caracteriza por tener umbrales de confianza social muy bajos, que resienten toda la estructura de relación con extraños.

Según el estudio de Valenzuela y Cousiño la desconfianza puede ser percibida como un rasgo cultural característico de 
nuestra sociedad, donde se percibe una desigualdad entre lo que se dice y se hace, situación que se contrasta con el predominio de la confianza en países desarrollados o países que han quebrado las premisas y contornos de la sociedad industrial y han podido abrir vías a una modernidad, denominada modernidad reflexi$v a$, flexible y transformadora de las estructuras que suponen la existencia de una dirección clara de desarrollo (Beck, Giddens y Lash, 2001).

La confianza en los jóvenes:

- Confianza en las Instituciones:

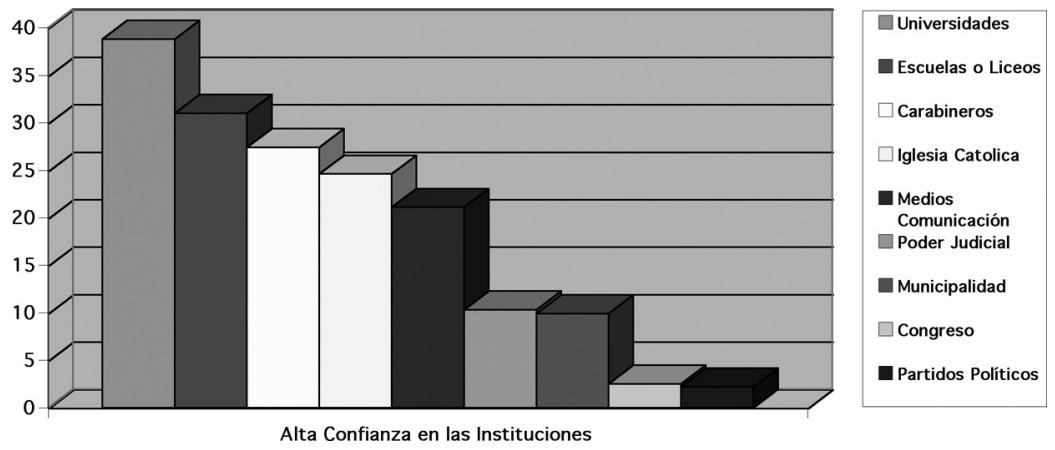

Según los datos de la VI Encuesta Nacional de Juventud, las instituciones educacionales (universidades y escuelas) son las que más confianza inspiran en los jóvenes, la que aumenta en la medida que disminuye la edad. Los partidos políticos y el Congreso son las instituciones que menos confianza le merecen a los jóvenes. Los jóvenes que viven en sectores rurales tienen más confianza en las instituciones en general

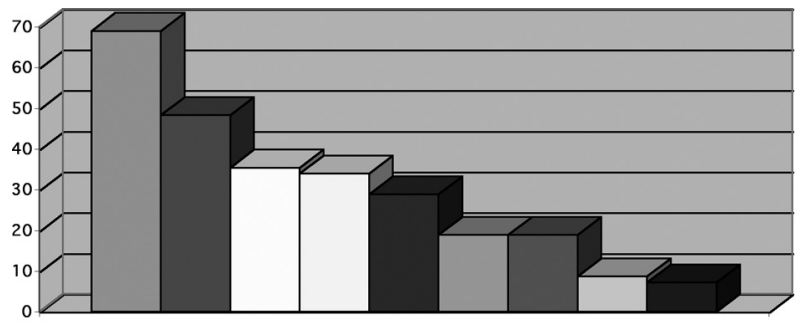

Baja Confianza en las Instituciones 
Los resultados mostrados por el gráfico anterior nos demuestran que los jóvenes tienen menos confianza en los partidos políticos (69\%), seguidos por el Congreso Nacional (48,3\%). El tercer lugar de desconfianza de los jóvenes lo ocupa del poder judicial, con un $35,4 \%$, y en cuarto lugar la Iglesia Católica con un 34\%.

\section{Confianza en las personas:}

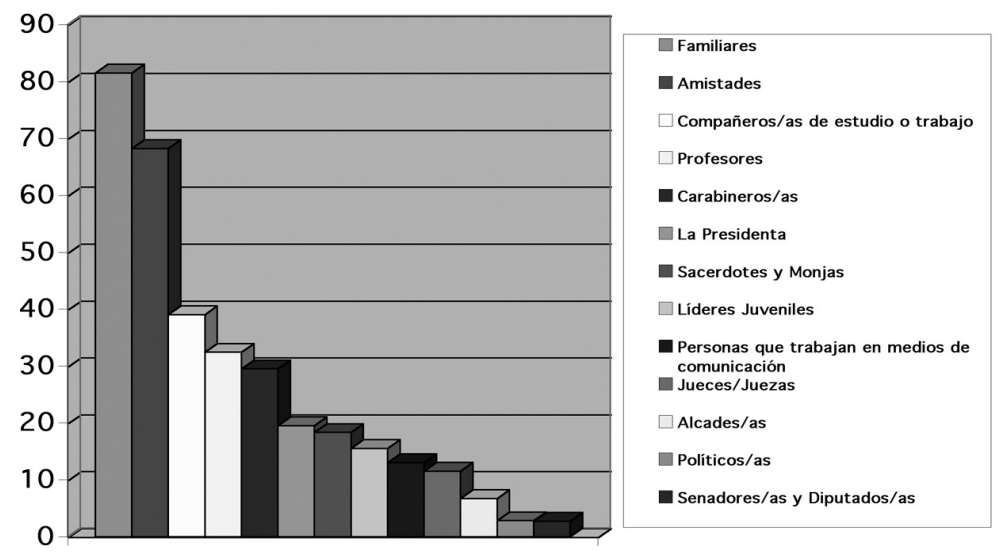

Alta Confianza en las Personas

La mayor confianza de los jóvenes está depositada en los familiares, con un $81,5 \%$, le siguen las amistades, con un $62,8 \%$. El tercer lugar en la confianza de los jóvenes está ocupado por los compañeros de trabajo o de estudio (39\%), seguidos por los profesores con un $32,4 \%$ y en quinto lugar los carabineros con un $29,5 \%$ de confianza.

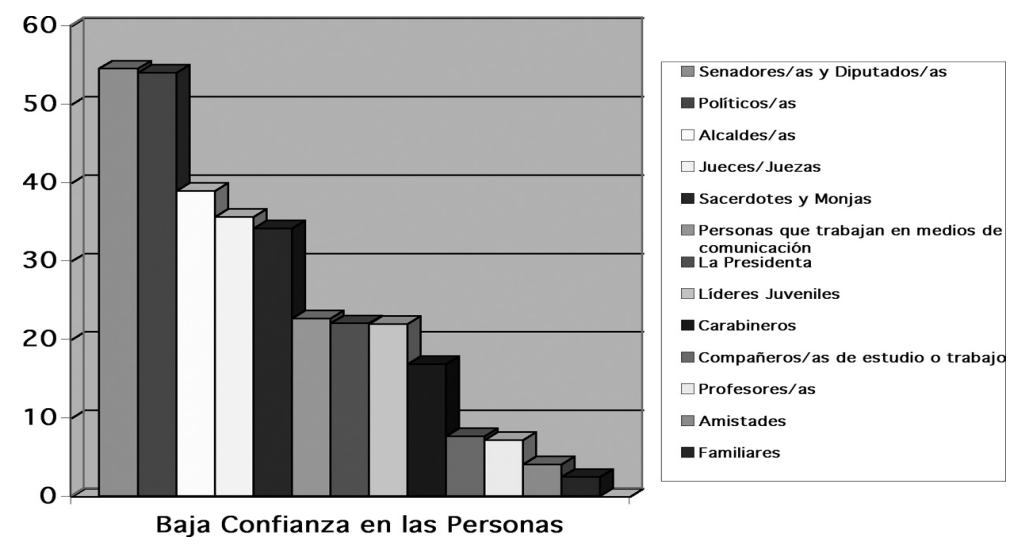


Las personas que menos confianza le merecen a los jóvenes son los Senadores (as) y Diputados (as), con un 54,5\%, en el segundo lugar de desconfianza están los políticos (as), con un $54 \%$, le siguen los Alcaldes con un 38,9\%. En cuarto lugar de desconfianza juvenil están los Jueces, con un 35,6\% y luego los Sacerdotes y Monjas, con un 34,1\% de desconfianza.

Los jóvenes tienen niveles de confianza similares tanto hacia las instituciones como hacia las personas que las integran. A pesar de ello prima una tendencia a confiar más en las personas que en las instituciones; es el caso del profesorado vs. Escuela/ Liceo, carabineros vs. Institución de carabineros, los jueces vs. poder judicial, los parlamentarios vs. El Congreso Nacional y los políticos vs. los Partidos Políticos, en ese mismo orden. Escapan a la tendencia la Iglesia, los medios de comunicación y los municipios, donde la institución tiene mayores porcentajes de confianza que las personas.

Por su parte, en el estudio realizado por Centro de Estudios en Juventud (CEJU), de la Universidad Católica Silva Henríquez sobre "valores en estudiantes secundarios", el año 2008, los resultados relacionados con la confianza de los jóvenes fueron los siguientes:

\section{Nivel de Confianza}

\begin{tabular}{|l|c|}
\hline & Porcentaje \\
\hline a. Que se puede confiar en la mayoría de la gente & $7,7 \%$ \\
\hline b. Que nunca se sabe las intenciones de los otros & $77,9 \%$ \\
\hline c. Que es mejor no confiar en nadie & $14,4 \%$ \\
\hline
\end{tabular}

Como se puede apreciar, la mayoría de los encuestados $(77,9 \%)$ plantea en términos generales que "nunca se sabe sobre las intenciones de los otros", tendiendo a no confiar totalmente en los demás. El porcentaje que confía en los demás es bajísimo, sólo un $7,7 \%$. Estos datos vienen a refrendar los hallazgos de las investigaciones del PNUD donde nos señalan que los chilenos operan básicamente por la desconfianza. Por su parte hay un 14,4\% que señaló directamente que es mejor no confiar en los otros. 
Cuando las personas o comunidades no confían en sus semejantes ni en las instituciones comienza a surgir la percepción de que no hay valores compartidos, por lo mismo no se confiará en la democracia ni en la igualdad social, perturbando el orden político e incluso el orden económico, los cuales constituyen el mundo social.

Lo anterior no quiere decir que la confianza en Chile no exista, se apunta a que existe pero de manera bastante discreta, siendo mínima en comparación a estándares internacionales. Se prefiere confiar en lo que se conoce, teniendo cautela ante lo desconocido. Según Valenzuela y Cousiño (2000), todos estos son hábitos que están muy arraigados en nuestra sociedad. Sobre lo mismo, indica Martínez (2001) que la desconfianza interpersonal "es un rasgo cultural característico de nuestra sociedad, lo que contrasta con el predominio de la confianza interpersonal en países desarrollados o de modernidad reflexiva" (p. 64).

Este panorama se evidencia en la Encuesta Mundial de Valores (MORI 2002, en Fundación Chile Unido, 2002), la que señala que nuestra sociedad es por naturaleza desconfiada, lo que se evidencia en el porcentaje de personas que manifiestan que "nunca es suficientemente cuidadoso en el trato con los demás" $(75 \%)$, versus quienes señalan que "se puede confiar en la mayoría de las personas" (22\%).

Esta situación se ha mantenido estable desde el año 1990 cuando se realizó el primer estudio en este aspecto, y se aleja mucho de los países desarrollados, donde el porcentaje de personas que piensa que se puede confiar en los otros bordea el $80 \%$.

Según E. Tironi (2008) el país latinoamericano donde más hay confianza es Argentina con 23\% y el menor es Brasil con apenas $3 \%$, cifra notoriamente baja en comparación con los países como Suecia (64\%), Estados Unidos (36\%), Alemania (33\%), Gran Bretaña (29\%) o Francia (21\%).

Otro espacio donde la confianza se ha visto cuestionada, es el 
educativo. En la Escuela, la confianza poco a poco ha dejado de ser un pilar en el cual se sostiene la comunidad educativa, Maturana (2006) señala que este medio de socialización tan medular, está aceptando los movimientos de desconfianza, donde se pierde el ser responsable y colaborador. Por lo mismo, los chilenos poseen un temor y miedo desmedido a lo desconocido, truncando sus relaciones más honestas con extraños.

De alguna forma, esta situación nacional es producto de la carga histórica que se arrastra como sociedad, de aquella historia reciente del país. E. Faletto (2009), en una entrevista acerca de una nueva ética del comportamiento realizada el año 2002, señala que las relaciones de confianza y de credibilidad sufrieron un revés durante la dictadura militar, en donde las condiciones dieron lugar para dudar de todo y de todos -de los vecinos, de los amigos, de las fuerzas de protección nacional, de los representantes políticos, etc.-, creándose "una retracción hacia un individualismo feroz, desconfianza con todo lo que viniera; oportunismo por todos lados" (p. 376).

\section{La Cohesión Social:}

La cohesión es definida por el Diccionario de la Real Academia Española como "la fuerza o acción mediante la cual los individuos pertenecientes a una sociedad se mantienen unidos". Así considerada, Palma (2008, p. 13) se pregunta: “¿cuál es esa fuerza o acción que permite a los individuos permanecer unidos?, y por otra parte: ¿cómo se genera esa fuerza?".

Desde la perspectiva sociológica, para la CEPAL (2007, p. 14) el concepto de cohesión social hace referencia "al grado de consenso de los miembros de un grupo social sobre la percepción de pertenencia a un proyecto o situación común".

La pregunta por la cohesión social (Tironi, 2006) hunde sus raíces en la sociología, puesto que autores como Durkheim, Weber, Simmel y Marx coinciden en que "la modernidad, a través del capitalismo, la individuación y la burocracia elimina las for- 
mas comunitarias y produce otras formas de integración; este proceso de transformación fue enmarcado por el paso de una cohesión estructurada en los vínculos primarios y las relaciones cara a cara, a otra caracterizada por la impersonalidad y las relaciones institucionalizadas".

Diversos escritos sobre cohesión social coinciden que uno de los principales planteamientos desde la sociología clásica, remiten de E. Durkheim, quien señala que cuanto menor es la división del trabajo en las sociedades, mayor es la vinculación de los individuos con el grupo social. En este sentido, el tema de la cohesión es parte de la solidaridad social necesaria para que los miembros de la sociedad sigan vinculados a ella. Sin embargo, el mismo Durkheim nos advierte que "la división social del trabajo que adviene con la modernización, erosiona y debilita tales vínculos, al igual que la creciente autonomía que adquiere el individuo en la sociedad moderna" (CEPAL, 2007, pp. 14-15).

Para Sorj y Tironi (2008, p. 87) la cohesión social asume características propias en cada sociedad y momento histórico, por ello, debe dar cuenta de las especificidades de cada sociedad. Por cuanto, "el problema de la cohesión social no es sólo un tema a estudiar; es también un problema a constituir".

Desde la configuración semántica del término cohesión social, se encuentra "la diferencia que reside en la dialéctica entre integración e inclusión, por una parte, y entre capital social y ética social, por otra" (CEPAL, 2007, pp. 14-15). De esta manera, se establece una "diferencia entre inclusión social y cohesión social, en la medida que la segunda incorpora la disposición y el comportamiento de los actores, aunque sin reducirse a ello".

Hopenhayn (2006) señala que la cohesión social se opone a la pérdida de normas de convivencia y estructuración social (anomia). A su vez, Sorj y Tironi (2008, p. 106) plantean que la cohesión social "se construye, se desarrolla y, si todo sale bien, se estabiliza en el tiempo; pero también se agota y, muchas veces, se quiebra". 
Para Sorj y Tironi (2008) el enfoque sociológico del término cohesión social, incluye las dimensiones sociales de la familia, el mercado y la sociedad civil. A su vez, el tema de la cohesión social incluye sentimientos de solidaridad con fines comunes, como son: confianza, asociatividad, acceso equitativo a la información, tolerancia, interculturalidad, entre otras.

Desde la ética social, se alude al concepto de cohesión social como "la comunidad de valores, el consenso en torno a mínimos normativos y mínimos sociales, la solidaridad como valor ético y valor práctico y un principio asumido de reciprocidad en el trato" (CEPAL, 2007. p. 16).

La cohesión social hace referencia a "los comportamientos y valoraciones de los sujetos que forman parte de la sociedad, abarcando ámbitos tan diversos como la confianza en las instituciones, el capital social, el sentido de pertenencia y solidaridad, la aceptación de normas de convivencia y la disposición a participar en espacios de deliberación y en proyectos colectivos" (CEPAL, 2007, p. 15).

Ello permitiría vincular dimensiones de la realidad distanciadas entre sí como son la política social y el valor de la solidaridad difundido en la sociedad; las sinergias entre equidad social y legitimidad política; la transmisión de destrezas y el empoderamiento de la ciudadanía; las transformaciones socioeconómicas y los cambios en la interacción social; los cambios socioeconómicos y los cambios en la subjetividad colectiva; la promoción de una mayor igualdad y de un mayor reconocimiento de la diversidad (sea esta de género, etnia o raza), las brechas socioeconómicas y el sentido de pertenencia.

En la noción de cohesión social subyace el concepto de capital social, concebido como "patrimonio simbólico de la sociedad en términos de la capacidad de manejo de normas, redes y lazos sociales de confianza, que permiten reforzar la acción colectiva y sentar bases de reciprocidad en el trato, que se extienden progresivamente al conjunto de la sociedad (...) acervo de los agen- 
tes sociales que contribuye a una sociedad más cohesionada" (CEPAL, 2007, pp. 15-16).

Otro componente de la cohesión social es la noción de integración social, entendida como "el proceso dinámico y multifactorial que posibilita a las personas participar del nivel mínimo de bienestar que es consistente con el desarrollo alcanzado en un determinado país" (CEPAL, 2007, pp. 15-16).

En este sentido, Palma (2008) establece que cuando se habla de cohesión social se está considerando la inclusión social o la generación de igualdad de oportunidades, pero también otros elementos; estableciendo que la cohesión social se desarrolla en cinco dimensiones que son: sentido de pertenencia, inclusión, participación, reconocimiento y legitimidad; y que estas dimensiones tienen expresión continua en su valoración positiva, que expresaría cohesión, y su expresión negativa, que denotaría falta de cohesión social. Así, un eje o dimensión varía de pertenencia a aislamiento; otro de inclusión a exclusión, un tercero de participación a no consideración, el cuarto de reconocimiento hacia rechazo y el último se desarrolla desde la legitimidad hacia la ilegitimidad.

Los procesos de cohesión social, desde Alonso (2002, p. 4), quien cita a Pierre Bourdieu, señala que "son procesos conflictivos de lucha por un capital simbólico -la ciudadanía reconocida, en gran parte conformada por una posición laboral estable- que los grupos dominantes tratan de controlar y bloquear en su acceso y por el que los grupos dominados pugnan en estrategias ya sean de adaptación solidaria e individualizada o de confrontación y acción colectiva".

En el contexto actual de globalización, la noción sobre cohesión social sugiere por un lado un "anhelo de comunidad perdida" (Hopenhayn 2006), con la consiguiente "falta de certezas para unir a las personas en torno a ideales compartidos"; y por otro lado, plantea una urgencia de gobernabilidad democrática ante la agudización de brechas salariales y sociales, la dispersión de intereses y demandas y el exceso de individuación. 
En este sentido, Sorj y Tironi (2008) plantean que la cohesión social suele evocar un anhelo de comunidad ante un escenario de globalización y transformaciones profundas, que muchos asocian con una mayor fragmentación social y una pérdida de lazos estables.

Sobre el uso contemporáneo del concepto, desde Sorj y Tironi (2008) se disemina a partir de las elaboraciones provenientes de la Unión Europea. La visión europea señala que la cohesión social es la capacidad de una sociedad de asegurar el bienestar de todos sus miembros -comunidad de individuos libres que se apoyan en la búsqueda de estos objetivos comunes bajo medios democráticos- y tiene como referencia la "cultura de derechos sociales".

En relación con la realidad de América Latina, tenemos que ocurre algo distinto con el tema de la cohesión social, puesto que "se ha sustentado básicamente en el plano de la cultura y la reciprocidad, con elementos como la comunidad, el mestizaje, la religiosidad, las relaciones familiares, el patronazgo, el caciquismo, el populismo, entre otros, ocupando un lugar más central que el Estado, la sociedad civil o el mercado, al menos si se le compara con las trayectorias de Europa o los Estados Unidos" (Sorj y Tironi, 2008, pp. 118-119).

En América Latina históricamente se intentó replicar una cohesión social de tipo europeo, basada primordialmente en la acción del Estado y Chile no fue la excepción. Hubo desde siempre voces críticas que resistieron apelando a ciertos rasgos específicos de un ethos cultural latinoamericano. No obstante, para Palma (2008) "lo que ha prevalecido en el caso de Chile es, más bien, el reemplazo de la antigua matriz europea por un nuevo marco de cohesión social de corte estadounidense en cuyo centro están la propiedad, el mercado y la sociedad civil".

Según Tironi, en Chile (2006, p. 49) históricamente "la cohesión social se basó en patrones europeos, y éstos han sido sustituidos por una nueva matriz fundada en el modelo estadounidense". 
En este sentido, "las nuevas generaciones han hecho suyos los valores individualistas que se promueven en todas las áreas de la vida. Aunque las preguntas sobre la integración comunitaria, la vida familiar, el bienestar personal, la protección del medio ambiente, la desigualdad o la cohesión social comienzan a hacerse con mayor frecuencia; y las falencias del modelo estadounidense son conocidas. La ética individualista puede llevar a la obtención de mayor prosperidad material, pero reduce la vida familiar, el contacto con los amigos, la sensación de pertenencia y hasta los índices de felicidad".

La cohesión social surge como un desafío ante las transformaciones de las sociedades contemporáneas, la CEPAL (2007) señala que la "corrosión de la legitimidad y gobernabilidad de los Estados nacionales, la acentuación de las brechas sociales, el surgimiento de identidades autorreferidas, la excesiva racionalización económica y la tendencia, también excesiva, a la individualización y el debilitamiento de lo público" (p. 13) son los contravalores que motivan poder hacer algo para evitar la materialización de la comunidad perdida.

Igualmente, E. Tironi (2008) afirma que "en el último periodo las sociedades latinoamericanas han experimentado un giro dramático respecto de lo que fuera la matriz histórica que las caracterizó durante gran parte del siglo XX (...) esta gran transformación ha erosionado ciertas relaciones sociales de tipo tradicional sobre las que reposaba históricamente la cohesión social en muchos países de la región, sin ofrecer alternativas inclusivas de reemplazo" (p. 11).

En ese proceso surge el concepto de cohesión social, como herramienta de convergencia social para socavar los males sociales de estos tiempos, y Güell señala, asimismo, que la cohesión social es uno de los puntos que se deben favorecer para que los cambios culturales obtengan el sentido necesario para su consolidación ciudadana (Güell, 2008).

Es por ello que las premisas de la cohesión social son la participación política y la confianza interpersonal e institucional; si un 
ciudadano aporta a fortalecer la cohesión social, es un ciudadano activo, al contrario, si solo demanda derechos, es un ciudadano pasivo. Y de aquí es que se entiende que la cohesión social no solo significa un desafío ante niveles de exclusión, sino que va más allá de ser integración social, es acción y evolución ciudadana.

La cohesión social promueve la solidaridad y las responsabilidades comunes de una sociedad al tiempo que baja la vulnerabilidad social, ya que si la construcción de la confianza potencia el "nosotros", es éste el que se manifiesta también vinculado a la cohesión social, llevando a la idea de protección mutua (Hirschman, en CEPAL, 2007, 24). En este sentido, la cohesión social motiva un tipo de protección a futuro, la cual debe darse de manera contextualizada, tomando en cuenta las singularidades de cada país para que sea realmente efectiva.

Un dato de relevancia es que tanto la cohesión social como la confianza social es uno de los fundamentos de la sociedad liberal. Sociedad liberal que por medio de la cohesión social logra importantes resultados, pero que en Chile "nunca se ha vivido realmente" (Valenzuela y Cousiño, 2000).

Fukuyama (1996) plantea que la cohesión social contribuye a la formación de capital social. Él sostiene que existen algunas sociedades más proclives a la asociación voluntaria, las cuales sacan al individuo del seno parental, a diferencia de otras sociedades donde la familia es la que cumple las funciones de asociación manteniendo fuertes lazos y el arraigo íntimo. De esta forma se puede comprender cómo, según el autor, el primer tipo de capital social facilita la cohesión social y el segundo visualiza una sociedad más hermética desde sus bases.

Por otro lado, la cohesión social motiva el progreso y el crecimiento económico de un país. Este aspecto se puede observar y es mucho más concreto en las sociedades que fomentan los tipos de asociaciones voluntarias que se distancian de las relaciones demasiado familiares, ya que abre las puertas a otros agentes 
extraños para que puedan operar en su economía, no así en las tradiciones familistas, donde hay más restricciones (Fukuyama, 1996). Se incita, desde este punto, a dar el salto a economías más amplias.

En la XVII Cumbre Iberoamericana Chile 2007 (2007, p. 10) se señala que disminuir la corrupción y mejorar la estabilidad política resultan fundamentales para la paz social y la sana convivencia entre las personas. "Cuando las relaciones humanas son vistas en un plano de cooperación, confianza y estabilidad, se asegura una mejor cohesión social y desarrollo general del país. Con más libertad se cumplen mejor esos objetivos. Bajo sistemas libres mejoran las oportunidades educacionales, la oferta de salud y las posibilidades de empleo, factores fundamentales para el desarrollo humano de los países, en paz y armonía".

Desde una perspectiva psicosocial la cohesión es lo que le da vida al grupo, manteniéndolo unido y proporcionando mayor interacción, influencia social, productividad y satisfacción entre sus miembros. Entonces tenemos que este enfoque se encuentra en la línea de la conducta grupal y no proporciona una visión amplia en cuanto a la interacción a nivel macro social de los individuos.

Ahora bien, si tomamos el planteamiento de la CEPAL (2007), que define como eje central de la cohesión social el sentido de pertenencia a la sociedad, en el que se conjugan elementos como percepciones, valoraciones y disposiciones de quienes integran la sociedad; y si a ello le agregamos la dimensión desde la ética social de la cohesión social de Hopenhayn (2006), que en términos subjetivos señala que es "la interiorización por parte de los sujetos de un 'saber conducirse' en las relaciones con los demás, donde los bienes públicos y el bienestar general aparecen como motivaciones que guían comportamientos de las personas" y en términos objetivos se refiere a la comunidad de valores, el consenso en torno a mínimos normativos y mínimos sociales, la solidaridad como valor ético y valor práctico, y un principio asumido de reciprocidad en el trato. 
Promover la cohesión social (Palma, 2008) requiere, entre otras cosas, "generar y fortalecer un sentido de pertenencia ligado en primera instancia al barrio en que se vive, así como a la comunidad más amplia, generando a su vez igualdad de oportunidades, implica considerar que los actores sociales son sujetos de transformación de su realidad, en la medida en que tienen la posibilidad de comprender y modificar las problemáticas que les afectan, reproduciéndose y sedimentándose en la cultura e identidad local".

El sentido de pertenencia depende de diversos factores y es promovido desde distintas instancias, a través del fortalecimiento de lo común. Ello se concreta por ejemplo, en el uso y cuidado de espacios comunes como la ciudad y el medio ambiente; el acuerdo respecto de ciertos valores de convivencia, tales como la tolerancia ante la diversidad y la reciprocidad en el trato; una participación más amplia en instancias de deliberación y en la expresión pública de aspiraciones y visiones de mundo; el combate a la violencia; la humanización de los principales espacios de socialización y el acceso difundido a los productos culturales.

Es así como la CEPAL (2007) considera un cruce entre ciudadanía y pertenencia, ello supone la intersección entre derechos sociales instituidos y solidaridad social internalizada. Ello encuentra sentido en la cohesión social a través del fortalecimiento de la disposición de los actores a ceder beneficios, en aras de reducir la exclusión y la vulnerabilidad de grupos en peores condiciones. Esta significación va más allá del valor ético, puesto que también toma un valor práctico, ya que mientras mayor sea el progreso en el ámbito de los derechos sociales y mayor es la solidaridad colectiva.

Desde el ámbito local, Gordon y Millán (2009), establecen un registro de cohesión social a partir de campos analíticos sobre las bases con las que se sustenta y los elementos que la conforman, a saber: 
a) Las pautas de unidad que prevalecen sobre una comunidad, constituidas por la confianza, las normas de reciprocidad y la capacidad de controlar el ambiente en que se vive;

b) Los vínculos sociales dados por la participación en organizaciones de carácter formal;

c) Los vínculos sociales establecidos en la sociabilidad informal;

d) Las redes sociales, su extensión, su estructura y sus prácticas de intercambio, $y$

e) La cooperación.

\section{Conclusiones:}

La familia no es un lugar donde la confianza nazca de forma espontánea sino que se desarrolla gradualmente, se demarca y limita desde un comienzo. Al interior de la familia se ponen límites y generan espacios de comunicación, lo cual permite que las relaciones humanas se construyan desde la confianza.

El vínculo social implica cierta complejidad para las personas, ya que se debe tener en cuenta una serie de reglas y normas que se deben respetar para entablar una adecuada interacción. A su vez se debe considerar que la concepción del mundo como un todo con las entidades individuales que existen en él nos lo presenta como una complejidad difícil de manejar (Luhmann, 1996), donde los seres humanos experimentan diversas sensaciones y emociones al encontrarse con otro ajeno a su vida social cotidiana pudiendo ser capaz de realizar comportamientos solidarios de cooperación o bien, comportamientos egoístas o individualistas, el que adopte uno u otro será a causa del riesgo que genera la interacción y el impedimento de ejercer control sobre una situación aumentando la incertidumbre. Generar y desarrollar confianza en las interrelaciones e intercambios entre las personas proporcionará seguridad en cada relación, disminuyendo la incertidumbre que surge en cada nueva reciprocidad. 
Por tanto, la confianza se da dentro de un marco de interacción que está influenciado tanto por la personalidad como por el sistema social, y no puede estar asociado exclusivamente con uno y otro.

La confianza se encuentra ligada al control y al poder predecir las supuestas intenciones de los demás, es por esto que una promesa se interpreta como información acerca de la conducta futura y que permite reducir la incertidumbre, por ende, la confianza es requerida para la reducción de un futuro caracterizado por una complejidad más o menos indeterminada.

Se confía para fantasear respecto a la seguridad que tendremos sobre futuras acciones, donde resulta necesario tener control sobre éstas porque pueden llegar a poner en peligro el presente.

Actualmente existe una necesidad por mantener el control, proponiendo normas externas para regular todo tipo de transacciones; para realizar cualquier tipo de transacción comercial se deben firmar documentos y para confiar en que la persona es quien dice ser, se han instaurado artefactos electrónicos donde se coloca la huella digital para comprobar su identidad. El valor de la palabra se ha perdido de la sociedad contemporánea.

La palabra de una persona ya no es suficiente para confiar, ahora es necesario que sea demostrado y si se descubre algún tipo de traición el individuo es castigado. Sin embargo este tipo de medidas no aportan a que existan mayores niveles de confianza, por el contrario se potencia la desconfianza y una constante vigilancia y duda; actualmente vivimos la cultura de la sospecha.

En este contexto se tienden a potenciar los logros individuales más que los grupales reforzando la confianza en el propio esfuerzo y desconfianza en las acciones que realizan los demás y desvalorizando el trabajo en equipo y la participación en la sociedad. Por lo mismo los bajos índices de participación, en el ámbito social y/o político, se pueden explicar por la desconfianza de los 
chilenos a unirse con extraños para el cumplimiento de metas comunes.

La reciprocidad, la cooperación y confianza son elementos que dan contenido al capital social y constituyen un importante recurso sociocultural en cualquier grupo humano, sin embargo, todos los datos encontrados nos señalan que la sociedad chilena actual transita en la dirección contraria, es decir, las interacciones sociales cotidianas se basan en la desconfianza, en la sospecha y la incertidumbre, afectando de manera significativa la posibilidad real de fortalecer el tejido social y por consecuencia la cohesión social entre los chilenos.

El ejercicio de la confianza social como preámbulo para una cohesión social fortalecida, ayuda a la estabilización de los vínculos interpersonales y los vínculos con las instituciones y organizaciones. Las sociedades necesitan vínculos. No se pueden manejar desde la sola individualidad de las personas, al contrario, para construir democracia, para volver a lo público y para incentivar el respeto por los Derechos Humanos, se torna un requisito necesario la confianza social, añadiendo un tipo de cohesión social que tome en cuenta la diversidad. Solo bajo estas virtudes sociales de corresponsabilidad y unión, la sociedad puede comenzar a dar fruto desde lo colectivo, incrementando grados de satisfacción que superan el éxito individual, pues apuntan a resaltar la grandeza de las personas en su acción social.

Chile se caracteriza por tener una baja cohesión social, ya que la gente percibe que la acción colectiva no solo es difícil, sino que tiene baja incidencia en la construcción de la sociedad.

Lo anterior conlleva el impulso a la racionalización de las aspiraciones colectivas como ideales posibles de alcanzar pero por el conjunto de la población, siendo todos beneficiados de la misma meta conseguida. La construcción de aspiraciones supone la existencia de confianza en la capacidad de acción, propia o ajena, para realizarlas. Esto es lo que distingue a las aspiraciones de 
la simple fantasía, significa también que las aspiraciones son un puente entre la subjetividad presente y el proyecto futuro, entre los deseos individuales y el orden colectivo que se espera.

Finalmente es necesario subrayar que si bien la confianza no es la única variable que contribuye al desarrollo de una sociedad sana con vínculos sociales estables, sí es una variable imprescindible. La confianza reduce la complejidad social yendo más allá de la información disponible y generalizando las expectativas de conducta en que se reemplaza la información que falta con una seguridad internamente garantizada. De este modo, es dependiente de otros mecanismos de reducción desarrollados paralelamente con ésta, por ejemplo los de la ley, de la organización y por supuesto, los del lenguaje, sin embargo no puede reducirse a ellos. Por lo tanto, la confianza es transversal a cualquier tipo de proceso, de decisión, de manifestación, de todo el quehacer de sujetos que se relacionan con otros de manera permanente.

Recuperar la confianza es inundarnos de aire puro, es respirar en una atmósfera que no impera en el actual estado de cosas de la sociedad chilena. Fraternidad y confianza deberían ser las consignas inscritas en las políticas sociales, las que lleven a la elevación de una nueva sociedad enriquecida de ella misma, la que da paso a la vitalidad cultural del país. Asimismo, la confianza en conjunto con la memoria y la autonomía, deben asumirse como los canales hacia una acción social de impacto. La confianza sobrepasa los niveles económicos o políticos, para instalarse de manera transversal en la matriz cultural chilena.

\section{Bibliografía:}

ALONSO, Luis (2002). Centralidad del Trabajo y Cohesión Social: ¿Una Relación Necesaria? Universidad Autónoma de Madrid. En Internet http://www.unavarra.es/puresoc/pdfs/lealonso1.pdf

ALLPORT, Gordon (1986). La personalidad: su configuración y desarrollo. Editorial Herder.

CEPAL (2007). Cohesión social: inclusión y sentido de pertenencia en América Latina y el Caribe. CEPAL; Agencia Española de Cooperación 
Internacional; Secretaría General Iberoamericana. LC/G.2335, Enero, Naciones Unidas, Santiago de Chile. Extraído en junio de: http://www.oei.es/quipu/cohesion_socialAL_CEPAL.pdf

COVEY, Stephen y Merrill, Rebecca (2007). El Factor Confianza: El Valor que lo Cambia Todo. Editorial Paidós Empresa. Nueva York, Barcelona.

DUBET, Francois (2009). Le travail des societés. Éditions du SEUIL. París, Francia.

ERIKSON, Erik (1987). Infancia y sociedad. Editorial Paidós. Buenos Aires, Argentina.

FALETTO, Enzo (2009). Dimensiones sociales, políticas y culturales del desarrollo. Siglo del Hombre Editores, Clacso, Bogotá. Extraído en junio de: http://bibliotecavirtual.clacso.org.ar/ar/libros/coedicion/faletto/

FUKUYAMA, Francis (1996). Confianza. Las virtudes sociales y la capacidad de generar prosperidad. Editorial Atlántida, $4^{a}$ Edición, Buenos Aires.

GARCÍA, Gonzalo (2001). La Transición a la Democracia: ¿Un proceso de confianza política? En Gobierno de Chile. "Confianza social en Chile. Desafíos y proyecciones". Unidad de Investigación y Desarrollo, División de Organizaciones Sociales, Ministerio Secretaría General de Gobierno. Santiago de Chile.

GOBIERNO de Chile (2001). Confianza social en Chile. Desafíos y proyecciones. Unidad de Investigación y Desarrollo, División de Organizaciones Sociales, Ministerio Secretaría General de Gobierno. Santiago de Chile.

GORDON, Sara y MILLÁN, René (2009). Análisis preliminar de la cohesión social. Un estudio comparativo. Cuadernos de investigación / Universidad Nacional Autónoma de México, N 41, UNAM, México.

GÜELL, Pedro (2008). Chile: Entre el autoritarismo y la expansión cultural de la ciudadanía. En Rojas, S.; Eisenberg, A.; Güell, P.; Araya, R.; Caetano, G.; Estévez, F.; Bravo, L.; Roumeau, J.; Álvarez, J.; Carrasco, M.; Yutronic, J.; Quevedo, V.; Ponczner, M.; Wayne, M.; Jarpa, V.; Navarro, A. y Martínez, A. “Ciudadanía, participación y cultura". Editorial LOM, Santiago de Chile, Primera edición.

HERREROS, Francisco (2004). ¿Por qué Confiar? Formas de Creación de Confianza Social. En Revista Mexicana de Sociología, Año 66, Núm. 4.

HOPENHAYN, Martín (2006). Cohesión social: Una Perspectiva en Proceso de Elaboración. En Cohesión Social en América Latina y el Cari- 
be: Una Revisión Perentoria de Algunas de sus Dimensiones. CEPAL-Colección Documento de proyecto.

INSTITUTO Nacional de la Juventud (2009). Sexta Encuesta Nacional de Juventud; principales resultados. Gobierno de Chile.

Jocelyn-Holt, Alfredo (2001). Un Dudoso llamado a la "Confianza": Una Historia Ambigua. En Confianza Social en Chile, Desafíos y Proyecciones. Gobierno de Chile, División de Organización Sociales. Santiago de Chile.

KEIM, Marcel (2001). La confianza en Chile. Antecedentes y desafios para una Cultura Democrática. Extraído desde: Gobierno de Chile. "Confianza social en Chile desafíos y proyecciones", pp. 58-67 Santiago - Chile.

KLIKSBERG, B \& Tomassini, L. comp. (2000). Capital social y cultura: claves estratégicas para el desarrollo. Buenos Aires-Argentina.

LASO, Esteban. Confianza, Desarrollo y Corrupción: Un Estudio de Psicología Social. Ponencia FLACSO 2007. En Internet http:/ / estebanlaso. com/pdfs/ponenciaflacso.pdf

LUHMMAN, Niklas (1996). Confianza. Primera edición Editorial Anthopos, en coedición con Universidad Iberoamericana y con Instituto de Sociología de la Pontificia Universidad Católica de Chile. España.

MARTÍNEZ, Marcelo (2001). La Confianza en Chile. Antecedentes y desafíos para una Cultura Democrática. En Gobierno de Chile. "Confianza social en Chile. Desafios y proyecciones". Unidad de Investigación y Desarrollo, División de Organizaciones Sociales, Ministerio Secretaría General de Gobierno. Santiago de Chile.

MATURANA, Humberto (2006). Educación desde la matriz biológica de la existencia humana. Biología del conocer y biología del amar. En Carrasco, E.; Negrón, B. y Astorga, A. (editores), "Sentidos de la educación y la cultura: cultivar la humanidad". LOM Ediciones, Santiago.

MONTAÑ́́S, Darío (s/f). La clave está en la confianza; taller de sociología de la Universidad de Cantabria. Extraído el 16 de julio del 2010 desde: http://centros3.pntic.mec.es/cp.cisneros/confianza.htm

MOYA, Luis (2001). Lo mágico del confiar: Datos de la política. En busca de la política. En busca de una fenomenología de la Confianza Social. En Gobierno de Chile. "Confianza social en Chile. Desafíos y proyecciones". Unidad de Investigación y Desarrollo, División de Organizaciones Sociales, Ministerio Secretaría General de Gobierno. Santiago de Chile. 
MOULIAN, Tomás (2001). Desconfianza ontológica y confianza relacional. En Gobierno de Chile. "Confianza social en Chile. Desafíos y proyecciones". Unidad de Investigación y Desarrollo, División de Organizaciones Sociales, Ministerio Secretaría General de Gobierno. Santiago de Chile.

OLEA, Francisco (2001). (Pre) Texto político: Entre la confianza y la incertidumbre. En busca de una fenomenología de la Confianza Social. En Gobierno de Chile. "Confianza social en Chile. Desafíos y proyecciones". Unidad de Investigación y Desarrollo, División de Organizaciones Sociales, Ministerio Secretaría General de Gobierno. Santiago de Chile.

PALMA, Andrés (2008). Las Políticas Públicas que no Contribuyen a la Cohesión Social. Fundación Internacional y para Iberoamérica de Administración y Políticas Públicas (FIIAPP).

PNUD (2000). Desarrollo Humano en Chile 2000. Más Sociedad para Gobernar el Futuro. Programa de las Naciones Unidas para el Desarrollo. Más sociedad para gobernar el futuro", p. 136. Extraído desde: Gobierno de Chile (2001) "Confianza social en Chile desafíos y proyecciones", p. 73, Santiago - Chile. Extraído en junio de: http:/ / www.desarrollohumano.cl/

PUTNAM, Robert (1993). Making Democracy Work: Civic Traditions in Modern Italy. Princeton: Princeton University Press.

PUTNAM, Robert; Pharr, Susan J. and Dalton, Russell J. (2000). Introduction: What's Troubling the Trilateral Democracies?, en Disaffected Democracies, editado por Susan J. y Putnam Pharr, Robert. Princeton, New Jersey: Princeton University Press.

PUTNAM, Robert (2002). SÓLO EN LA BOLERA. Colapso y surgimiento de la comunidad norteamericana. Barcelona: Galaxia.

ROJAS, Francisco (1994). Un proceso de construcción de confianza. En Varas, A. y Caro, I. (Editores), "Medidas de confianza mutua en América Latina". FLACSO, Santiago.

ROJAS, Francisco (1996a). Introducción: la verificación como herramienta determinante en la construcción de confianza. En Rojas, F. (editor), "Medidas de confianza mutua: verificación". FLACSO, Santiago.

ROJAS, Francisco (1996b). Balance estratégico y medidas de confianza mutua. En Rojas, F. (editor), "Medidas de confianza mutua: verificación". FLACSO, Santiago.

ROJAS, Francisco (1996c). Medidas de confianza mutua y balance estratégico: un vínculo hacia la distensión y la estabilidad. En Rojas, F. (editor), 
"Balance estratégico y medidas de confianza mutua". FLACSO, Santiago.

SALAZAR, Gabriel (2001). Proyecto y Exclusión: dialéctica histórica de la desconfianza en Chile. En Gobierno de Chile. "Confianza social en Chile. Desafíos y proyecciones". Unidad de Investigación y Desarrollo, División de Organizaciones Sociales, Ministerio Secretaría General de Gobierno. Santiago de Chile.

SORJ, Bernardo y Tironi, Eugenio (2008). Cohesión Social en América Latina: Un Marco de Investigación. Proyecto Nueva Agenda de Cohesión Social en América Latina, elaborado por el CIEPLAN y el Instituto Fernando Henrique Cardoso.

TIRONI, Eugenio (2006). Cohesión Social en Chile: El Retorno de un Viejo Tema. En revista Quórum, Nº 18. Santiago de Chile.

V Encuentro del Grupo de Biarritz (2009). Cohesión Social y Gobernabilidad en América Latina. Documento de discusión. Julio 19 y 20 de 2009. Ciudad de Guatemala, por la Corporación Escenarios de Colombia.

VALENZUELA, Eduardo y COUSIÑO, Carlos (2000). Sociabilidad y asociatividad. Un ensayo de sociología comparada. Eduardo Valenzuela y CEP, Estudios Públicos: No 77. Extraído en junio de: http:// www.cepchile.cl/dms/lang_1/doc_1220.html

XVII Cumbre Iberoamericana Chile 2007. Libertad: La Mejor Receta Para La Cohesión y La Oportunidad Social. En Internet http:/ / www.lyd. com/lyd/controls/neochannels/neo_ch3756/deploy/cumbre.pdf 